\title{
The utility of gallium-68 DOTATOC PET/CT in lymphangioleiomyomatosis
}

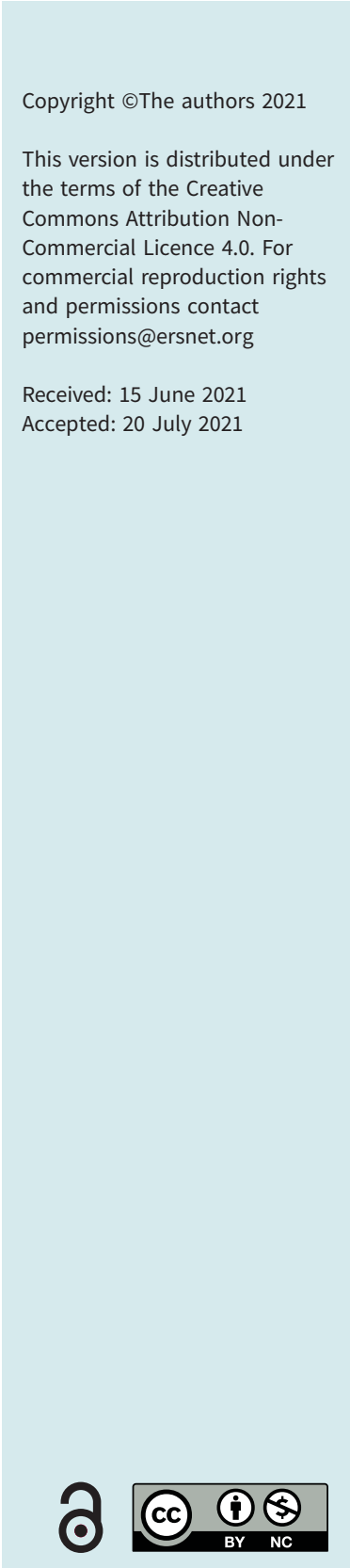

To the Editor:

Lymphangioleiomyomatosis (LAM) is a progressive, low-grade, metastasising neoplasm of women that is characterised by infiltration of the lung parenchyma with abnormal smooth muscle-like cells, resulting in cystic lung destruction [1]. While forced expiratory volume in $1 \mathrm{~s}\left(\mathrm{FEV}_{1}\right)$ is the current standard outcome measure used in LAM, there are limitations to its use as a true surrogate of disease severity and it may underestimate disease burden. Additionally, pulmonary function testing depends on patient cooperation and there can be significant intertest variation. Despite vascular endothelial growth factor (VEGF)-D being an excellent diagnostic test and biomarker of prognosis [2], it is not elevated in all patients; hence, other biomarkers of disease activity and prognosis are required. Due to the neoplastic nature and the activation of mechanistic target of rapamycin (mTOR) signalling in LAM, it has previously been investigated whether LAM lesions would demonstrate increased metabolic uptake of $\left[{ }^{18} \mathrm{~F}\right] 2$-fluoro-2-deoxyglucose (FDG) on positron emission tomography (PET) scanning. However, no significant uptake of FDG was demonstrated in LAM lesions or angiomyolipomas (AMLs), and this radiotracer was not deemed suitable to determine disease burden or activity in sporadic or tuberous sclerosis-associated LAM (TSC-LAM) patients [3]. Patients with LAM may develop chylous complications, including chyloptysis, chylous ascites and chylothorax [4, 5]. Additionally, TSC-LAM can be associated with neuroendocrine tumours that overexpress somatostatin receptors, and octreotide, a somatostatin analogue, is an effective therapy for chylothorax in a variety of conditions, including in LAM [6, 7]. While it is not known whether somatostatin receptors are expressed in LAM lesions, it is known that somatostatin and urotensin II are closely related neuropeptides, and directly activate both urotensin receptors and somatostatin receptors [8]. Urotensin II and urotensin receptors are present and expressed to a greater extent in the lungs of LAM patients compared to normal lungs [9]. In prior reports, octreotide scintigraphy demonstrated increased uptake of radiolabelled octreotide diffusely throughout the lungs and kidneys, suggesting the presence of somatostatin receptors or analogous receptors in LAM [10]. Gallium-68-linked somatostatin receptor PET radiotracers such as ${ }^{68} \mathrm{Ga}$-DOTA-TyI ${ }^{3}$-octreotide (DOTATOC) $\left({ }^{68} \mathrm{Ga}\right.$-DOTA PET/computed tomography (CT)) utilise a radiolabelled somatostatin analogue peptide that binds with high affinity to the somatostatin receptors. ${ }^{68} \mathrm{Ga}$-DOTA PET/CT offers many advantages over octreotide scintigraphy, with significantly higher target-to-background ratio, superior spatial resolution, better sensitivity and potential for simpler quantification [11]. We hypothesised that ${ }^{68} \mathrm{Ga}$-DOTA PET/CT could be a useful molecular imaging technique in LAM that could demonstrate disease burden.

Following local institutional review board approval (reference number RS20-014), patients were recruited from the LAM clinic at St Vincent's University Hospital (Dublin, Ireland) and provided informed written consent. Sporadic LAM patients were selected that were not on mTOR inhibitor therapy. Age- and sex-matched control patients were included from the national neuroendocrine service who had ${ }^{68} \mathrm{Ga}$-DOTA PET/CT with normal lung parenchyma.

All ${ }^{68} \mathrm{Ga}$-DOTA-chelated somatostatin analogue peptide PET/CT examinations were performed on a Siemens Biograph mCT PET-CT system (Siemens Healthineers, Forchheim, Germany). A standard PET acquisition from skull base to upper thighs was acquired after injection of $122.6 \pm 12.6 \mathrm{MBq}$ of ${ }^{68} \mathrm{Ga}$-DOTATOC with an average tracer uptake time of $61 \pm 5 \mathrm{~min}$. Non-attenuation-corrected and attenuation-corrected datasets were reconstructed. The low-dose, unenhanced CT component was performed with patients maintaining normal shallow respiration, using a standardised protocol with $140 \mathrm{kV}$, pitch 1.375 and automatic milliamperes (15-100 mA, noise index 40). CT images were

Shareable abstract (@ERSpublications)

Somatostatin receptor functional imaging is of limited utility as an imaging biomarker in LAM, but other PET/CT modalities may be of use https://bit.ly/3l6BVZp

Cite this article as: Gaffney B, Lynn E, Dodd JD, et al. The utility of gallium-68 DOTATOC PET/CT in lymphangioleiomyomatosis. ERJ Open Res 2021; 7: 00397-2021 [DOI: 10.1183/23120541.00397-2021]. 

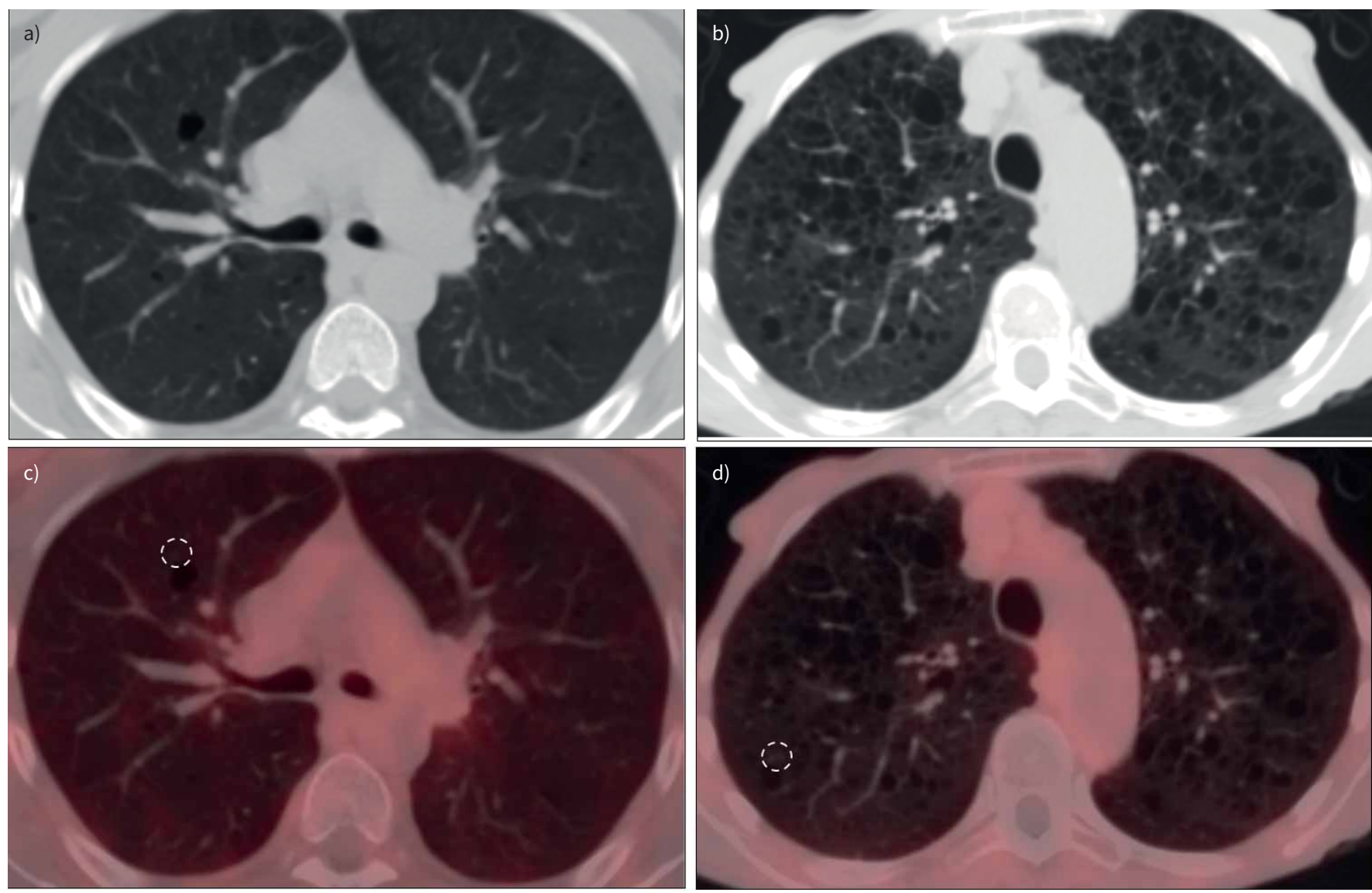

Right upper lobe

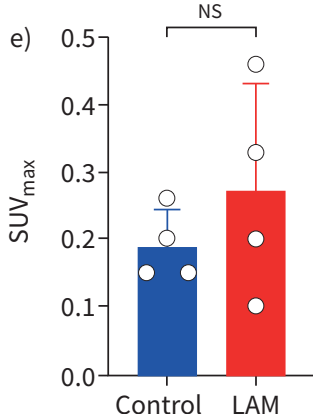

Right upper lobe

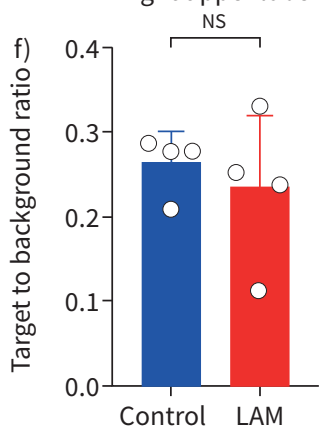

Right middle lobe

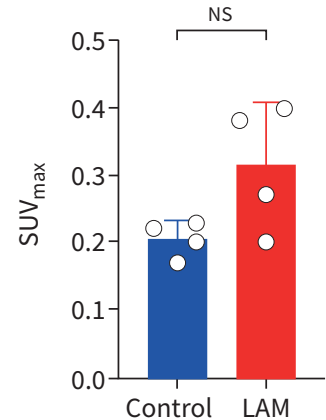

Right middle lobe

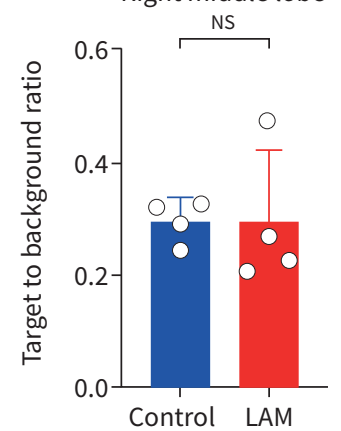

Right lower lobe

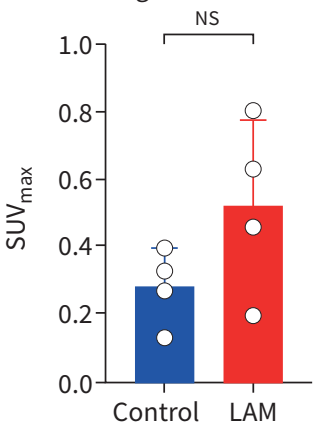

Right lower lobe

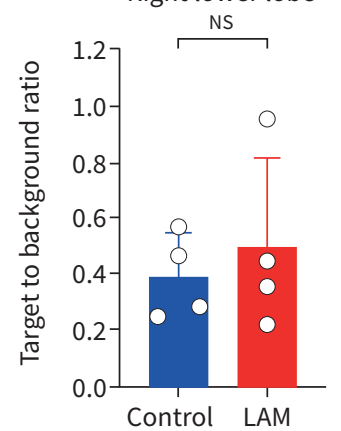

Left upper lobe
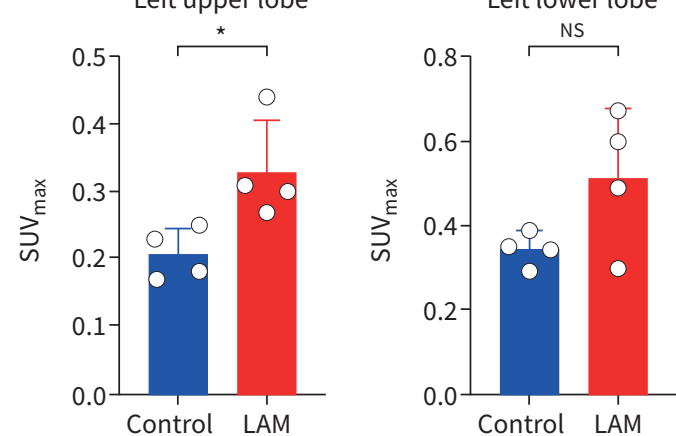

Left upper lobe

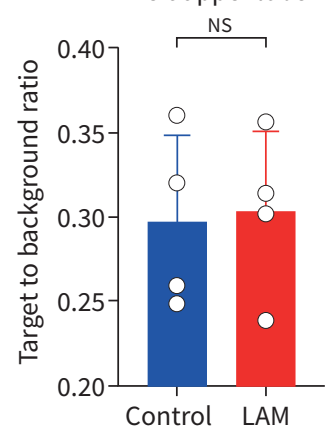

Left lower lobe

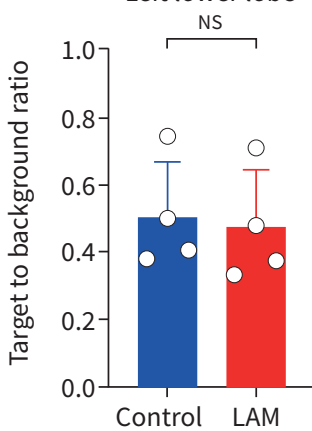

FIGURE 1 Qualitative and quantitative assessment of ${ }^{68} \mathrm{Ga}$-DOTA-Tyl ${ }^{3}$-octreotide (DOTATOC) positron emission tomography (PET)/computed tomography (CT) in lymphangioleiomyomatosis (LAM). a) Axial thoracic CT image at the level of the carina in a patient with mild LAM (forced expiratory volume in $1 \mathrm{~s}\left(\mathrm{FEV}_{1}\right): 99 \%$ of predicted; diffusing capacity of the lung for carbon monoxide $\left(D_{\mathrm{LCO}}\right): 88 \%$ of predicted) demonstrates 
occasional scattered thin-walled cysts. c) No increased tracer uptake evident on the axial fused ${ }^{68}$ Ga-DOTATOC PET/CT image (maximum standardised uptake value $\left(\mathrm{SUV}_{\max }\right)$ window range $\left.0-5\right)$. b) A separate patient with severe $\mathrm{LAM}\left(\mathrm{FEV}_{1}: 36 \%\right.$ of predicted; $D_{\mathrm{LCO}}: 25 \%$ of predicted) has innumerable cysts demonstrated on this axial thoracic CT image at the level of the aortic arch. d) No increased pulmonary tracer uptake evident on ${ }^{68} \mathrm{Ga}$-DOTATOC PET/CT. Dashed line: sample region of interest demonstrated on PET images panels $c$ and d. e) Semiquantitative SUV max measures of pulmonary ${ }^{68} \mathrm{Ga}$-DOTATOC uptake in lungs of patients with LAM and controls. f) Target to background ratio for each lobe calculated by dividing the lobar SUV max $_{\text {max }}$ by the mediastinal blood pool SUV $\max$ in lungs of patients with LAM and controls. Each dot represents an individual patient. Data are presented as mean \pm SD. LAM, $n=4$; control; $n=4$. Ns: nonsignificant. *: $p<0.05$, unpaired Student's t-test.

reconstructed with a slice thickness of $2.5 \mathrm{~mm}$. A standard clinical PET time-of-flight ordered subset expected maximisation reconstruction using two iterations, 24 subsets and a Gaussian filter was performed. PET images were reconstructed with a slice thickness of $2.5 \mathrm{~mm}$ and pixel size of $4 \mathrm{~mm}$.

Qualitative and semiquantitative analysis was performed in a random order, blinded to the clinical information. Qualitative global assessment of pulmonary ${ }^{68} \mathrm{Ga}$-DOTATOC uptake was performed using a three-point visual scale: 1, less than mediastinal blood pool (MBP); 2, greater than MBP but less than liver; 3 , greater than liver. Semiquantitative maximum standardised uptake value $\left(\mathrm{SUV}_{\max }\right)$ measures of pulmonary ${ }^{68} \mathrm{Ga}$-DOTATOC uptake were performed in each lobe by drawing a region of interest (ROI) of $\sim 1 \mathrm{~cm}$ in each lobe. For patients with LAM, the ROI was drawn in cyst-adjacent parenchyma. A freehand ROI was outlined in the arch of the aorta, avoiding the aortic walls, to define $\mathrm{SUV}_{\max } \mathrm{MBP}$. A target to background ratio (TBR) was calculated for each lobe by dividing the lobar $\mathrm{SUV}_{\max }$ by MBP SUV $\mathrm{Max}_{\max }$.

Four female patients aged 49, 53, 55 and 82 years, with LAM, underwent whole-body ${ }^{68}$ Ga-DOTA PET/ CT. Three of the patients were post-menopausal and one perimenopausal. The median diffusing capacity of the lung for carbon monoxide $\left(D_{\mathrm{LCO}}\right)$ was $59.75 \%$ of predicted, median $\mathrm{FEV}_{1}$ was $58.5 \%$ of predicted and median VEGF-D was $641 \mathrm{pg} \cdot \mathrm{mL}^{-1}$. There was no qualitative difference in tracer uptake in any region of the lung or extrathoracically in LAM patients compared to controls, with all studies having a score of 1 (i.e. less than MBP) (figure 1a-d) Moreover, there was no significant difference in the overall mean lobar $\mathrm{SUV}_{\max }$ of LAM lungs except in the left upper lobe (0.33) compared to control $(0.2075)(\mathrm{p}=0.0275)$; however, this is unlikely to be of any significant importance (figure 1e). Crucially, there were no differences in the TBR in any lobe of LAM patients compared to controls (figure 1f), indicating that there was no significant uptake of ${ }^{68}$ Ga-DOTATOC in the lungs of patients compared to controls. There were no significant correlations between mean $\mathrm{SUV}_{\max }$ or TBR values and pulmonary function measures in patients with LAM. Of note, there were incidental findings noted of moderate hydronephrosis in one patient requiring intervention and a pulmonary nodule that required surveillance.

The results of this pilot study indicate that there is no demonstrably increased uptake of ${ }^{68} \mathrm{Ga}$-DOTATOC in LAM and that somatostatin receptor analogue functional imaging is unlikely to be of utility as an imaging biomarker in this disease. Despite the small number of studies, the severity of LAM varied in this group. The $D_{\text {LCO }}$ ranged from $25 \%$ to $110 \%$ of predicted. Three LAM patients had AMLs, with two having undergone surgical removal. No patient was on rapamycin at the time of imaging, although one patient had been previously but stopped due to side-effects and two patients have since been commenced on rapamycin. Two patients required supplemental oxygen. There were no significant differences in radiotracer uptake, indicating a lack of signal for this modality. This current study using ${ }^{68} \mathrm{Ga}$-DOTA PET/CT suggests that earlier proposals that there is somatostatin receptor-bearing tissue in LAM is likely to be incorrect and that treatment with octreotide is unlikely to be of benefit through any direct mechanism [10]. This is important to report as it excludes this imaging approach or other somatostatin molecular imaging modalities in LAM in the future. Other functional imaging approaches may be of benefit in the future, such as ${ }^{68}$ Ga-NEB PET/CT [12] or carbon-11-labelled glutamine PET/CT, designed to assess whether increased glutamine uptake in LAM lesions, previously demonstrated in mechanistic preclinical studies of LAM, has utility as a clinically meaningful biomarker [13]. Further studies are required to identify improved diagnostic and prognostic approaches in LAM. Brian Gaffney $\oplus^{1,2}$, Evelyn Lynn ${ }^{1}$, Jonathan D. Dodd ${ }^{2,3}$, Michael P. Keane $e^{1,3}$, David J. Murphy ${ }^{2,3}$ and
Cormac McCarthy ${ }^{1,3}$

${ }^{1}$ Dept of Respiratory Medicine, St Vincent's University Hospital, Dublin, Ireland. ${ }^{2}$ Dept of Radiology, St Vincent's University Hospital, Dublin, Ireland. ${ }^{3}$ School of Medicine, University College Dublin, Dublin, Ireland. 
Provenance: Submitted article, peer reviewed.

Author contributions: C. McCarthy and D.J. Murphy conceived and designed the study. B. Gaffney, E. Lynn, M.P. Keane and C. McCarthy identified and recruited patients. D.J. Murphy and J.D. Dodd interpreted radiological imaging. All authors drafted the manuscript. C. McCarthy is the guarantor of the paper.

Conflict of interest: None declared.

Support statement: This study was funded by The LAM Foundation: grant number LAM0144SG01-20. Funding information for this article has been deposited with the Crossref Funder Registry.

\section{References}

1 Henske EP, McCormack FX. Lymphangioleiomyomatosis - a wolf in sheep's clothing. J Clin Invest 2012: 122: 3807-3816.

2 Young LR, Lee HS, Inoue Y, et al. Serum VEGF-D concentration as a biomarker of lymphangioleiomyomatosis severity and treatment response: a prospective analysis of the Multicenter International Lymphangioleiomyomatosis Efficacy of Sirolimus (MILES) trial. Lancet Respir Med 2013: 1: 445-452.

3 Young LR, Franz DN, Nagarkatte P, et al. Utility of $\left[{ }^{18} \mathrm{~F}\right] 2$-fluoro-2-deoxyglucose-PET in sporadic and tuberous sclerosis-associated lymphangioleiomyomatosis. Chest 2009: 136: 926-933.

4 Johnson SR, Tattersfield AE. Clinical experience of lymphangioleiomyomatosis in the UK. Thorax 2000: 55 : 1052-1057.

5 Ryu JH, Doerr CH, Fisher SD, et al. Chylothorax in lymphangioleiomyomatosis. Chest 2003: 123: 623-627.

$6 \quad$ Kalomenidis I. Octreotide and chylothorax. Curr Opin Pulm Med 2006: 12: 264-267.

7 Namba M, Masuda T, Nakamura T, et al. Additional octreotide therapy to sirolimus achieved a decrease in sirolimus-refractory chylous effusion complicated with lymphangioleiomyomatosis. Intern Med 2017: 56: 3327-3331.

8 Malagon MM, Molina M, Gahete MD, et al. Urotensin II and urotensin II-related peptide activate somatostatin receptor subtypes 2 and 5. Peptides 2008: 29: 711-720.

9 Kristof AS, You Z, Han YS, et al. Protein expression of urotensin II, urotensin-related peptide and their receptor in the lungs of patients with lymphangioleiomyomatosis. Peptides 2010: 31: 1511-1516.

10 Upadhyay DC, Corbridge T. Octreotide acetate uptake in tuberous sclerosis complex with lymphangioleiomyomatosis. Int J Nucl Med 2001; $1: 1$.

11 Johnbeck CB, Knigge U, Kjaer A. PET tracers for somatostatin receptor imaging of neuroendocrine tumors: current status and review of the literature. Future Oncol 2014: 10: 2259-2277.

12 Hou G, Xu W, Jiang Y, et al. Lymphangioleiomyomatosis revealed by ${ }^{68} \mathrm{Ga}$-NOTA-Evans Blue PET/CT. Eur J Nucl Med Mol Imaging 2020: 47: 2469-2470.

13 Hewlett J, Manning H, Young L, et al. PET imaging of glutamine uptake in lymphangioleiomyomatosis. Am Thorac Soc 2020; 201: A4972. 